Preprint: Article to be published in Public Library Quarterly

\title{
Using Open Data to Inform Public Library Branch Services
}

\author{
Karalyn R Ostler, Bree Norlander \& Nicholas Weber \\ Information School, University of Washington, Seattle, USA
}

1851 NE Grant Ln,

Seattle, WA 98105

Corresponding author: nmweber@uw.edu

Funding Details: This work was supported by the Institute for Museum and Library Services (IMLS) under the Grant - Open Data Literacy ( RE-40-16-0015-16).

\section{Biographical notes:}

Karalyn Ostler is a Master of Library and Information Science student at the University of Washington Information School. She was a 2019 Open Data Literacy Intern.

Bree Norlander is a Research Coordinator \& Analyst for the Open Data Literacy project and the Technology \& Social Change (TASCHA) Group at the University of Washington (UW). She received her M.L.I.S. from the UW iSchool specializing in Data Science and Data Curation. Her research focuses on curating, analyzing, and advancing Open Data.

Nicholas Weber is an Assistant Professor in the Information School at the University of Washington, and the co-Principal Investigator of the IMLS funded project Open Data Literacy. Dr. Weber's research focuses on the development, use, and maintenance of public interest technologies. 
Preprint: Article to be published in Public Library Quarterly

\section{Using Open Data to Inform Public Library Branch Services}

This article describes the curation and use of open demographic data to inform public library services. A case study of census data curated for the Seattle Public Library (SPL) system is described. To understand the information needs of library branches, a set of SPL regional managers were interviewed, a set of use cases were created, and a prototype dashboard tool using open census data was developed to address the needs of two SPL regions. The utility of available open data to meet the needs of regional managers is reviewed, as well as the potential development of replicable data analysis tools for keeping public libraries aware of shifting neighborhood demographics.

Keywords: Open Data, Data-driven decision making, Library system management. 


\section{Preprint: Article to be published in Public Library Quarterly}

\section{Introduction}

Public libraries are often described as a social infrastructure - serving as a key public institution where residents can turn for their information and technology needs (McAllen, Downs \& Ascan, 2017). As such, the need to understand and reliably respond to rapidly changing demographics is crucial for public libraries. In order to effectively serve constituents that may differ significantly by neighborhood throughout a large metropolitan region, public libraries need granular data and accessible analytic tools (Shepherd, Petrillo \& Wilson, 2019, Turner \& Gorichanaz, 2018) .

The branch system of large urban centers are already well positioned to cater to the unique needs of residents but understanding how and when those needs shift - based on the composition of residents - remains a challenging data analysis task (Shepherd, Petrillo \& Wilson, 2018; Allen, 2019). For example, many large public library systems employ some form of community profiling for understanding the uses and users of different branch outlets (Jaeger et al., 2014). This approach is typically based on Geographic Information System (GIS) applications that allow for the mapping of a service area using underlying socio-demographic data (Sharma, 2015). GIS-based tools are useful for many public library systems attempting to create visual depictions of users and their corresponding attributes (e.g. population, race, income). A notable example of a GIS approach was the Public Library Geographic Database ${ }^{1}$ - a highly curated collection of demographic data in GIS layers produced by researchers at Florida State University. This early example showed the promise of using freely accessible data from multiple sources to create useful visualizations to public library staff. However, highly curated databases require long-term maintenance that are often difficult without

\footnotetext{
${ }^{1}$ The Public Library Geographic Database was funded through IMLS and produced by researchers at Florida State University. This database has, however, not been updated since 2005. https://gisuser.com/2005/02/public-library-geographic-database-plgdb-mapping/
} 


\section{Preprint: Article to be published in Public Library Quarterly}

sustained funding to do so. In more recent years, online platforms such as the United States Census Bureau's data.census.gov ${ }^{2}$ provide data exploration tools for viewing publicly available demographic data. These online interfaces are useful for quickly viewing available data but can be difficult to navigate and customize. More advanced platforms like the Engagement Network ${ }^{3}$ created by the Center for Applied Research and Engagement Systems (CARES) at the University of Missouri takes exploring public data one step further, offering both public and custom tools for viewing similar demographic data. While the Engagement Network has a high level of customizability and reporting, these features extend beyond their free access and costs vary based on the level of customization. In addition to these tools, many other GIS solutions available to public library staff are proprietary, requiring extensive training on specific platforms that cannot be easily generalized (Arendt et al., 2018).

As part of the Open Data Literacy (ODL) project, a grant under the Institute for Library and Museum Services (IMLS) Laura Bush 21st Century Librarian program, we have been working to develop open-source solutions to help branches in the Seattle Public Library (SPL) system better understand the composition of their users, and how those demographics change over time. More broadly, the ODL project has focused on transforming MLIS curriculum at the University of Washington Information School to use open data as a means to connect students with public information service providers (Weber et al, 2018). Under the ODL grant we have also co-designed a paid summer internship program that places Information School graduate students with a public sector sponsor to work on publishing, promoting, or using open data to improve their service delivery. The first author on this paper spent a summer working at the Seattle

\footnotetext{
${ }^{2}$ Data.census.gov is the new platform for accessing data and digital content from the U.S. Census Bureau.

3 https://engagementnetwork.org/
} 


\section{Preprint: Article to be published in Public Library Quarterly}

Public Library to improve their use of open demographic data in designing outreach and service programs throughout the Seattle region. Based on this preliminary work, we believe that there is an opportunity to create simple dashboards using freely available census data that will help public libraries from any region to better understand and respond to the needs of particular neighborhoods without investing in expensive proprietary GIS tools to do so.

In the following paper, we describe the methods used to develop a prototype mapping dashboard that gives branch managers in the Seattle Public Library the ability to identify and query key demographic data about branch constituents. We first review the process of generating requirements from interviews with SPL staff and background research. We then describe potential open data sources available to meet their needs, and open-source software that can be used to analyze and produce visual representations of this data. We then present a prototype dashboard that can be easily maintained by non-expert software users. We conclude by outlining the potential for expanding this work by using a reproducible R notebook (2018) that can be modified and adopted by any public library.

\section{Background}

Public interest technology (PIT) development seeks to use openly available data and software to serve a communal interest (Michael, et al., 2019). As part of this growing technology movement, PIT holds great promise to improve service delivery for public facing institutions in cost-effective ways. In conceiving of a public library branch system as a complex and dynamic public service (Freeburg, 2018) we seek to use systems development methods from the field of Public Interest Technology to serve the 


\section{Preprint: Article to be published in Public Library Quarterly}

Seattle Public Library (SPL). Seattle is a dynamic and rapidly growing city that has in the past and continues to evolve its public services with respect to new and shifting demographics (Hillard et al., 2014). As large urban library systems, such as SPL, continue to innovate with service models for targeted populations (e.g. Wahler et al., 2019) we believe it is critical to understand how to best identify which neighborhoods need which services, and, crucially, how these needs change over time.

\section{Setting}

The Seattle Public Library is an urban branch system with 27 different branch locations that serve particular neighborhoods in the city of Seattle, WA. Each branch offers a variety of services and outreach programs based on the perceived needs of the surrounding neighborhood. Prior to this project, the primary approach to understanding neighborhoods surrounding each branch library was in the form of "community listening" - where librarians reach out to local organizations with strong relationships to marginalized populations, seeking information about possible needs that could be addressed by the library (Scott, 2011). This narrative form of learning works well for identifying general characteristics about a given community of stakeholders but is limited by the amount of time and personnel available to perform the interviews and analyze relevant data. To supplement these efforts, SPL has increasingly sought to use outside open data sources to create actionable intelligence for their frontline staff.

SPL had been partnering with ODL since its inception but had not engaged in a data science focused project with ODL interns prior to the project described in this article. In collaboration with an SPL Data Analyst this project followed best practices in public interest technology development including three main phases: an assessment of 


\section{Preprint: Article to be published in Public Library Quarterly}

staff data needs, a search for suitable open data, and the creation of visual materials for staff use. An overarching goal for this project, and any public interest technology, is to use open source materials and methods so that the outcomes could be reproducible, sustainable, and accessible. We conclude the article with a discussion of how our methods and software can be reused in new settings.

\section{Materials and Methods}

The following three subsections will cover the methods used in each of the three phases of this project. First, we will discuss the interviewing process that was followed to gather information about the information needs of library staff. Then, we describe the search for matching available open data and outline the steps needed to use data from the US Census Bureau. Finally, we explain the components of the interactive maps created for use by SPL staff, and how this work can be extended beyond the Seattle Public Library setting.

[ Place Image 1 here ]

Image 1: Map of Seattle Public Library branch outlets. The two regions that are the focus of this study include branches $3,6,13,14,18,19,20,22$, and 25 .

\section{SPL Staff Data Needs Assessment}

To develop a prototype of open data services for SPL we focused initially on two regions of Seattle that contain 9 total branch libraries (4 in the Northeast, and 5 in the Southeast). The Northeast and Southeast regions were selected based on the shifting demographics of underserved populations in these areas, and the general interest in using data by branch librarians at these outlets. Each semi-structured interview was 


\section{Preprint: Article to be published in Public Library Quarterly}

approximately 20 minutes in duration and conducted over the phone. In preparation for the interviews, a questionnaire was prepared with general information about the project and interviewer, a list of questions regarding general community information needs of SPL staff, and an inventory of potential open data sets.

Information from the interviews were transcribed and key emerging characteristics were synthesized into a list of common themes and then used to develop a set of 'use cases' that would guide the development and evaluation of our data analysis prototype. Use cases, and in particular users stories, in systems analysis and development are a human-centered approach to design. Use cases attempt to transform common systems requirements into functional specifications that a system designer can then build to directly support a user (Davis and Dawe, 2001; Wynholds et al., 2011).

From the semi-structured interviews, SPL regional managers expressed a need for more granular demographic data about the communities in proximity to each branch within their region. Previous demographic data gathering efforts at SPL had focused on larger parts of Seattle, providing statistics for the whole city, and specific regions instead of specific branches. To better understand which data we should curate for a prototype system we developed four user stories, centered around how regional managers, as key stakeholders, required information to assist in overall decisions for the region and how branch librarians might regularly use data for more specific service decisions at each branch. The following users stories, which follow the typical "As an X I want Y so that I can Z" format are offered as evidence of our synthesis:

User Story 1: As a Regional Manager, I want to know the age break-down of the population surrounding each of my branches so that I can determine what 


\section{Preprint: Article to be published in Public Library Quarterly}

type of services I need to be planning for in the next few years (i.e. youth services for children, resources for senior citizens).

User Story 2: As a Regional Manager, I want to know the ratio of kids going to public schools versus private schools in my region so that I can determine where I should be focusing my outreach services to best reach children who might lack additional education services.

User Story 3: As a Regional Manager, I want to know which parts of the community in my region have the lowest median income levels so that I can find places in the community that need career resources.

User Story 4: As a Branch Librarian, I want to know more information about my community so that I can better plan my services, like finding which parts of my community need certain resources and then providing outreach services for them.

Based on the needs of the interviewees, we chose four demographic characteristics to curate for our prototype development: age, median household income, language spoken at home, and K-12 school enrollment type. In the following section, we describe potential sources for this type of data, and which were ultimately selected for curation.

\section{Review of Open Data Sources}

Our search for relevant open data sets consisted of searching local and national open data repositories. We searched local open data portals including the City of Seattle 


\section{Preprint: Article to be published in Public Library Quarterly}

Open Data Portal ${ }^{4}$ and the Washington State Open Data Portal ${ }^{5}$, as well as data.gov ${ }^{6}$, a national repository of open data, and the United States Census Bureau ${ }^{7}$. Based on the factors collected from the interviews and derived use cases, we determined that the Census Bureau had the most relevant publicly accessible demographic data to meet our needs.

The Census Bureaus' American FactFinder allows users to search through the public data released by the Census Bureau's censuses and surveys ${ }^{8}$ and by using advanced search features results can be refined by topic, geography, and race and ethnic group. We identified five data sets from the 2017 American Community Survey (ACS) ${ }^{9}$ that closely reflected demographic information requested by regional managers ${ }^{10}$. Each data set provides estimated counts or averages for each census tract in King County. This data is meant to help local officials, community leaders, and businesses better understand their communities. ${ }^{11}$ For this project, we accessed the data needed for our dashboard development by using the Census Data application programming interface (API) through the R package tidycensus (Walker, 2019).

\section{Data Analysis and Visualization}

In order to analyze and present demographic data visually we chose to create an

\footnotetext{
${ }^{4} \underline{\text { https://data.seattle.gov/ }}$

${ }^{5}$ https://data.wa.gov/

${ }^{6} \mathrm{https}: / /$ www.data.gov/

${ }^{7} \mathrm{https}: / /$ www.census.gov/data.html

${ }^{8}$ As of July 2019, American Factfinder has been replaced by data.census.gov as the primary tool for accessing Census Bureau data and was decommissioned March 30, 2020.

${ }^{9} \mathrm{https}: / /$ www.census.gov/programs-surveys/acs/news/data-releases/2017/release.html

${ }^{10}$ The 5 datasets were: 1. Sex By Age, 2. Median Age By Sex, 3. Median Household Income In The Past 12 Months, 4. Age By Language Spoken At Home For The Population 5 Years And Over, and 5. Sex By School Enrollment By Level Of School By Type Of School For The Population 3 Years And Over 11 https://www.census.gov/programs-surveys/acs
} 


\section{Preprint: Article to be published in Public Library Quarterly}

interactive map ${ }^{12}$ with the programming language R's 'Shiny` package (Chang, Cheng, Allaire, Xie \& McPherson, 2019). R was chosen over other statistical computing and visualization languages for this project based on its open-source libraries for mapping, healthy development community for support, and because it was preferred by SPL data analysis staff. All R code created was saved in an R Markdown Notebook that can be reproduced, reused, and repurposed.

\section{[ Image 2 here]}

Image 2: Screenshot of Shiny dashboard.

To create the interactive maps with the R Shiny dashboard, we used the R package Leaflet (Cheng, Karambelkar, and Xie, 2018) which was optimal for this project because it allows users to create maps with multiple layers. By integrating a map of Seattle and the Census Bureau data we could visualize all 27 SPL branches. Four total maps, placed in separate tabs, were created covering median age, median household income, language spoken at home, and K-12 school enrollment by level of school. Each map consists of a base OpenStreetMap ${ }^{13}$ layer, a colored census tract layer, and a layer of branch location markers. Each census tract and library branch location also has a pop-up text bubble with the percentages of the community for each population group, allowing users to click around the map and see the general population breakdowns for each characteristic map. We chose pop-up information bubbles - a feature native to Shiny - to decrease the amount of data presented in a single map view while still

\footnotetext{
${ }^{12}$ To view app see https://kostler.shinyapps.io/SPL-Seattle-Census-Data/ ${ }^{13}$ OpenStreetMaps is the default base map for Leaflet and was chosen over other map layers because it was open source and provided ample geographic context for the other overlying layers: https://www.openstreetmap.org/
} 


\section{Preprint: Article to be published in Public Library Quarterly}

allowing a user to navigate and discover additional information within each map.

The Shinyapps.io platform was used to host the interactive web app with a stable URL for the Leaflet maps. Conveniently, Shiny converts the resulting visualizations from $\mathrm{R}$ code into a browser friendly application that retains interactivity and functionality while avoiding the clutter of code chunks that appear in an R notebook. The dashboard ${ }^{14}$ is available publicly, allowing any SPL staff member to use the information in their work.

\section{SPL Data Integration}

SPL, like many libraries, collects and analyzes patron data to evaluate programming, adjust services, and make purchasing decisions. This data often contains personally identifiable information that is difficult to de-identify or publish openly. However, for the purposes of internal analysis such data, when combined with more granular demographic data, could provide improved insight for making decisions at the branch and regional level of SPL. To explore this possibility, we acquired from our SPL partner, a dataset that included general counts of patron library card use by branch. We then stratified samples of this dataset into age groups (matching those strata that were available in the 2017 ACS data). Integrating these datasets allowed librarians to make meaningful heuristic evaluations of their services. For example, the population surrounding a branch (census data set) could be measured as a percentage of the population who are actually using the library (internal data set).

In doing this integration we found, as one would expect, a close relationship between the demographics of a branch and its surrounding neighborhoods. However,

\footnotetext{
${ }^{14}$ To view app see https://kostler.shinyapps.io/SPL-Seattle-Census-Data/
} 


\section{Preprint: Article to be published in Public Library Quarterly}

there were notable exceptions - for example in branches in SPL's Northeast region, the internal borrower dataset depicts a much higher population of youth using the branch than the population that census data reports living around the branch. Such discrepancies in use and population may have simple explanations - such as proximity to a school or bus stop. But, with easily aggregated statistics there is an opportunity to identify and explore these types of relationships - and where possible - adjust service offerings accordingly. In rapidly changing urban settings, like Seattle, we believe such analysis should be part of a trend towards data driven service development. There are a variety of other valuable datasets that could be used in conjunction with external open data sets to provide even more useful analysis of service offerings.

\section{Dashboard Implementation}

This project did not include a formal pilot period for the dashboard's implementation at SPL branches. The completed online dashboard URL and associated project summary reports were provided to the SPL regional managers and Data Analyst for distribution to branch librarians. With the provided URL, interested librarians can access the maps on any internet-enabled computer and do not need any coding experience or software downloaded to fully interact with the mapped data. The dashboard was designed for librarians to easily access the open Census Bureau data with minimal interaction with the $\mathrm{R}$ code that reads in the data directly from the Census Bureau API and then visualizes it as interactive maps. Librarians can easily integrate these data maps into their normal service planning and community research processes, like community listening, and can gain expanded insight on their patrons. The map can act as a starting point for finding significant characteristics of the community surrounding a library 


\section{Preprint: Article to be published in Public Library Quarterly}

branch and help determine next steps for developing services that best address the needs of those who need public libraries the most. For example, a branch librarian using the dashboard age map may find that the average age of people living around their branch is over 65 and after engaging with patrons then determine that more senior citizen programming is needed to best support the majority of the community who uses, or could use, that library branch.

In addition to providing a practical tool for viewing community demographic data, this project also highlighted the general interest in data among SPL staff and demonstrated what is possible for using open data to inform library services at SPL. Since creation of the prototype map dashboard, interest in open data and visualizations among SPL staff has increased greatly. After engaging with the dashboard and working with the ODL intern, library staff have continued to ask questions and create a wish list of data points that they would like to see visualized. As a result, several spin-off maps that build off the work of this project have been developed and used in outreach planning and internal advocacy work, including an internal "Equity Planning Map."

\section{Implications for Design}

This project prioritized the use of open source software and open data to create an exploratory demographic visualization of SPL branch service areas. Though the visualization dashboard was created to aid SPL staff, it is easily adaptable for other U.S. community organizations using census bureau data. All code used to build the interactive maps is structured in an R Markdown Notebook and includes documentation as well as instructive inline comments. With sufficient documentation detailing the code, other data professionals can use the same base code to produce customized maps 


\section{Preprint: Article to be published in Public Library Quarterly}

for their own geographic area. There are a number of other data visualization software options available for the activities described in this paper. While these options require less manual coding, they are often proprietary and require the purchase of a prohibitively expensive license. Open source software, like R, Rstudio, and all of the free code libraries that are developed for this language, are freely available for anyone to use.

The use of R also facilitates simple future updates by SPL with Census Bureau data. The Census Bureau releases all of its surveys and censuses in a standardized format, making it easy to update applications from year to year. Further, by using the Census API, our prototype could be easily updated by changing the year variable to the most recent release. Since census data will be structured the same as in previous years, the maps in our prototype can also be updated with minimal changes to the base code. This feature contributes to the sustainability of the dashboard. Each year, the dashboard will need to be updated but a majority of the base code will not need to be changed at all. The API also provides the possibility of eventually automating the updates, taking out the need for software updates altogether.

\section{Discussion and Conclusion}

Public libraries have access to the growing availability of open civic data on topics such as demographics, public health, weather, and more. Unfortunately, with the disparate nature of these datasets and the time and knowledge required to compile and map the data, most public libraries simply don't have the capacity to meaningfully engage with these valuable data sources. Proprietary tools do exist for subscription fees, but open government data is readily available for free analysis and use. Additionally, online 


\section{Preprint: Article to be published in Public Library Quarterly}

platforms provide free access to this data, but lack certain levels of customization. We have created a prototype of an open source application that uses freely available software and data to highlight local community demographics in specific Seattle neighborhoods. We have shown that libraries can gain an understanding of who lives within their areas and can compare this data with library-specific patron data to develop programs and services that best suit their community's needs.

This project also demonstrates how engaging library staff in data-related projects like this prototype map dashboard can help introduce library staff to open demographic data and increase interest in using open data to inform library services. From this prototype dashboard, SPL has already started to build more advanced maps upon the request of library staff. Now that staff have seen what is possible, they can continue to modify the products of this project and explore open data. By using the code developed for this project, other library systems only need to make slight modifications to recreate the dashboard for their own city and library system. Integrating open data into already existing library planning can help public libraries gain a better understanding of their communities and develop better services.

Our prototype explored demographic data from the American Community Survey. There is ample opportunity to advance this prototype by adding other datasets and testing their utility in creating programs and services. This research could be furthered in multiple ways such as interviewing additional library personnel offering different library service in different areas, adding new open government data sources, adding specific local library datasets, and evaluating the effectiveness of services created by analyzing this data. 
Preprint: Article to be published in Public Library Quarterly

\section{Declaration of Interest Statement}

The authors have no competing interests to declare

\section{References}

Allen, J. (2019). Mapping differences in access to public libraries by travel mode and time of day. Library \& Information Science Research, 41(1), 11-18.

Arendt, A., Morris, A., \& Stephens, M. (2018). Public Library Use of Geographic Information Systems in the United States. Journal of Library Administration, 58(8), $779-805$.

Chang, Winston; Cheng, Joe; Allaire, JJ; Xie, Yihui, and McPherson, Jonathan. (2019). shiny: Web Application Framework for R. R package version 1.3.2. https://CRAN.R-project.org/package=shiny

Cheng, Joe; Karambelkar, Bhaskar; and Xie, Yihui. (2018). leaflet: Create Interactive Web Maps with the JavaScript 'Leaflet' Library. R package version 2.0.2. https://CRAN.R-project.org/package=leaflet

Davis, L., \& Dawe, M. (2001, January). Collaborative design with use case scenarios. In Proceedings of the 1st ACM/IEEE-CS joint conference on Digital libraries (pp. 146-147). ACM.

Freeburg, D. (2018). Leadership and innovation within a complex adaptive system: Public libraries. Journal of Librarianship and Information Science, 0961000618810367. 
Preprint: Article to be published in Public Library Quarterly

Hillard, P., Love, L., Franks, H. M., Laris, B. A., \& Coyle, K. K. (2014). “They were only joking": Efforts to decrease LGBTQ bullying and harassment in Seattle public schools. Journal of school health, 84(1), 1-9.

Jaeger, P. T., Gorham, U., Bertot, J. C., \& Sarin, L. C. (2014). Public libraries, public policies, and political processes: Serving and transforming communities in times of economic and political constraint. Rowman \& Littlefield.

McAllen, D., Downs, G., \& Ascani, H. (2017, July). Shaping City Government Through Effective Technology Management: The Changing Role of Public Libraries. In 2017 Portland International Conference on Management of Engineering and Technology (PICMET) (pp. 1-3). IEEE.

Michael, K., Kobran, S., Abbas, R., \& Hamdoun, S. (2019, November). Privacy, Data Rights and Cybersecurity: Technology for Good in the Achievement of Sustainable Development Goals. In 2019 IEEE International Symposium on Technology and Society (ISTAS) (pp. 1-13). IEEE.

R Core Team. (2018). R: A language and environment for statistical computing. R Foundation for Statistical Computing, Vienna, Austria. URL https://www.R-project.org/. 
Preprint: Article to be published in Public Library Quarterly

Scott, R. (2011). The role of public libraries in community building. Public Library Quarterly, 30(3), 191-227

Sharma, D. M. (2015). Using GIS to assess public libraries. Public Libraries, 54(6), $19-20$.

Shepherd, J., Petrillo, L., \& Wilson, A. (2018). Settling in: how newcomers use a public library. Library Management.

Shepherd, J., Petrillo, L., \& Wilson, A. (2019). Does size matter? In-library study of two Canadian public library branches. Library Management.

Turner, D., \& Gorichanaz, T. (2018). Collaborative connections: Designing library services for the urban poor. The Library Quarterly, 88(3), 237-255.

Wahler, E. A., Provence, M. A., Helling, J., \& Williams, M. A. (2019). The changing role of libraries: How social workers can help. Families in Society. ISBN: 1044389419850707.

Walker, Kyle. (2019). tidycensus: Load US Census Boundary and Attribute Data as 'tidyverse' and 'sf'-Ready Data Frames. R package version 0.9.2. https://CRAN.R-project.org/package=tidycensus 
Preprint: Article to be published in Public Library Quarterly

Weber, N. M., Pamer, C. L., \& Norlander, B. (2018). Advancing open data: Aligning education with public sector data challenges. Proceedings of the Association for Information Science and Technology, 55(1), 927-928.

Wynholds, L., Fearon Jr, D. S., Borgman, C. L., \& Traweek, S. (2011, June). When use cases are not useful: Data practices, astronomy, and digital libraries. In Proceedings of the 11th annual international ACM/IEEE joint conference on Digital libraries (pp. 383-386). ACM. 